\title{
CERTAIN CLASS OF ANALYTIC FUNCTIONS WITH VARYING ARGUMENTS DEFINED BY THE CONVOLUTION OF SĂLĂGEAN AND RUSCHEWEYH DERIVATIVE
}

\author{
Á. O. PÁll-Szabó, O. Engel, E. Szatmári
}

ABSTRACT. In this paper we derive some results for certain new class of analytic functions with varying arguments defined by the convolution of Sălăgean and Ruscheweyh derivative and we study the properties of the image of this class through the Bernardi operator.

2010 Mathematics Subject Classification: 30C45.

Keywords: analytic functions, varying arguments, the convolution of Sălăgean and Ruscheweyh operator, extreme points, Bernardi integral operator.

\section{Coefficient estimates}

Let $\mathcal{A}$ denote the class of functions of the form:

$$
f(z)=z+\sum_{k=2}^{\infty} a_{k} z^{k}
$$

which are analytic and univalent in the open unit disc $U=\{z \in \mathbb{C}:|z|<1\}$.

Definition 1. [8]

For $f \in \mathcal{A}, n \in \mathbb{N}$, the Sălăgean differential operator $\mathscr{S}^{n}$ is defined by $\mathscr{S}^{n}: \mathcal{A} \rightarrow$ $\mathcal{A}$,

$$
\begin{gathered}
\mathscr{S}^{0} f(z)=f(z), \\
\mathscr{S}^{1} f(z)=z f^{\prime}(z), \\
\cdots \\
\mathscr{S}^{n+1} f(z)=z\left(\mathscr{S}^{n} f(z)\right)^{\prime}, z \in U
\end{gathered}
$$


Remark 1. If $f \in \mathcal{A}$ and $f(z)=z+\sum_{k=2}^{\infty} a_{k} z^{k}$, then

$$
\mathscr{S}^{n} f(z)=z+\sum_{k=2}^{\infty} k^{n} a_{k} z^{k}, z \in U
$$

Definition 2. [7]

For $f \in \mathcal{A}, n \in \mathbb{N}$, the operator $\mathscr{R}^{n}$ is defined by $\mathscr{R}^{n}: \mathcal{A} \rightarrow \mathcal{A}$,

$$
\begin{gathered}
\mathscr{R}^{0} f(z)=f(z), \\
\mathscr{R}^{1} f(z)=z f^{\prime}(z), \ldots \\
(n+1) \mathscr{R}^{n+1} f(z)=z\left(\mathscr{R}^{n} f(z)\right)^{\prime}+n \mathscr{R}^{n} f(z), z \in U .
\end{gathered}
$$

Remark 2. If $f \in \mathcal{A}$ and $f(z)=z+\sum_{k=2}^{\infty} a_{k} z^{k}$, then

$$
\mathscr{R}^{n} f(z)=z+\sum_{k=2}^{\infty} \frac{(n+k-1) !}{n !(k-1) !} a_{k} z^{k}, z \in U .
$$

Definition 3. [1] Let $n \in \mathbb{N}$. Denote by $\mathscr{S} \mathscr{R}^{n}$ the operator given by the Hadamard product (convolution) of the Sălăgean operator $\mathscr{S}^{n}$ and the Ruscheweyh operator $\mathscr{R}^{n}, \mathscr{S} \mathscr{R}^{n}: \mathcal{A} \rightarrow \mathcal{A}$,

$$
\mathscr{S} \mathscr{R}^{n} f(z)=\left(\mathscr{S}^{n} * \mathscr{R}^{n}\right) f(z), z \in U
$$

Remark 3. If $f \in \mathcal{A}$ and $f(z)=z+\sum_{k=2}^{\infty} a_{k} z^{k}$, then

$$
\mathscr{S} \mathscr{R}^{n} f(z)=z+\sum_{k=2}^{\infty} \frac{k^{n}(n+k-1) !}{n !(k-1) !} a_{k}^{2} z^{k}, z \in U .
$$

Definition 4. [5] Let $f$ and $g$ be analytic functions in $U$. We say that the function $f$ is subordinate to the function $g$, if there exists a function $w$, which is analytic in $U$ and $w(0)=0 ;|w(z)|<1 ; z \in U$, such that $f(z)=g(w(z)) ; \forall z \in U$. We denote by $\prec$ the subordination relation.

Definition 5. For $\lambda \geq 0 ;-1 \leq A<B \leq 1 ; 0<B \leq 1 ; n \in \mathbb{N}_{0}$ let $P(n, \lambda, A, B)$ denote the subclass of $\mathcal{A}$ which contain functions $f(z)$ of the form (1) such that

$$
(1-\lambda)\left(\mathscr{S} \mathscr{R}^{n} f(z)\right)^{\prime}+\lambda\left(\mathscr{S} \mathscr{R}^{n+1} f(z)\right)^{\prime} \prec \frac{1+A z}{1+B z} .
$$


Á. O. Páll-Szabó, O. Engel, E. Szatmári - Certain class of analytic functions ...

Attiya and Aouf defined in [3] the class $\mathscr{R}(n, \lambda, A, B)$ with a condition like (2), but there instead of the operator $\mathscr{S} \mathscr{R}^{n}$ they used the Ruscheweyh operator.

Definition 6. [10]

A function $f(z)$ of the form (1) is said to be in the class $V\left(\theta_{k}\right)$ if $f \in A$ and $\arg \left(a_{k}\right)=\theta_{k}, \forall k \geq 2$. If $\exists \delta \in \mathbb{R}$ such that $2 \theta_{k}+(k-1) \delta \equiv \pi(\bmod 2 \pi), \forall k \geq 2$ then $f(z)$ is said to be in the class $V\left(\theta_{k}, \delta\right)$. The union of $V\left(\theta_{k}, \delta\right)$ taken over all possible sequences $\left\{\theta_{k}\right\}$ and all possible real numbers $\delta$ is denoted by $V$.

Let $V P(n, \lambda, A, B)$ denote the subclass of $V$ consisting of functions $f(z) \in P(n, \lambda, A, B)$.

\section{MAin RESUlts}

Theorem 1. Let the function $f(z)$ defined by (1) be in $\mathrm{V}$. Then $f(z) \in V P(n, \lambda, A, B)$, if and only if

$$
T(f)=\sum_{k=2}^{\infty} k^{n+1} C_{k}(1+B)\left|a_{k}\right|^{2} \leq B-A,
$$

where

$$
C_{k}=[n+1+\lambda(k-1)(n+k+1)] \frac{(n+k-1) !}{(n+1) !(k-1) !} .
$$

The extremal functions are:

$$
f(z)=z+\sqrt{\frac{B-A}{k^{n+1} C_{k}(1+B)}} e^{i \theta_{k}} z^{k},(k \geq 2) .
$$

Proof. We work based on the technique used in [6].

Suppose that $f(z) \in V P(n, \lambda, A, B)$. Then

$$
h(z)=(1-\lambda)\left(\mathscr{S} \mathscr{R}^{n} f(z)\right)^{\prime}+\lambda\left(\mathscr{S} \mathscr{R}^{n+1} f(z)\right)^{\prime}=\frac{1+A w(z)}{1+B w(z)},
$$

where

$$
w \in H=\{w \text { analytic, } w(0)=0 \text { and }|w(z)|<1, z \in U\} .
$$

From this we have

$$
w(z)=\frac{1-h(z)}{B h(z)-A} .
$$

Therefore $h(z)=1+\sum_{k=2}^{\infty} C_{k} k^{n+1}\left|a_{k}\right|^{2} z^{k-1}$ and $|w(z)|<1$ implies 
Á. O. Páll-Szabó, O. Engel, E. Szatmári - Certain class of analytic functions ...

$$
\left|\frac{\sum_{k=2}^{\infty} C_{k} k^{n+1}\left|a_{k}\right|^{2} z^{k-1}}{(B-A)+B \sum_{k=2}^{\infty} C_{k} k^{n+1}\left|a_{k}\right|^{2} z^{k-1}}\right|<1 .
$$

Since $f(z) \in V, f(z)$ lies in the $V\left(\theta_{k}, \delta\right)$ for some $\left\{\theta_{k}\right\}$ sequence and a real number $\delta$ such that $2 \theta_{k}+(k-1) \delta \equiv \pi(\bmod 2 \pi), \forall k \geq 2$.

Set $z=r e^{i \delta}$ in (5), then

$$
\left|\frac{\sum_{k=2}^{\infty} C_{k} k^{n+1}\left|a_{k}\right|^{2} r^{k-1}}{(B-A)-B \sum_{k=2}^{\infty} C_{k} k^{n+1}\left|a_{k}\right|^{2} r^{k-1}}\right|<1 .
$$

Since $\Re\{w(z)\}<|w(z)|<1$ we have

$$
\Re\left\{\frac{\sum_{k=2}^{\infty} C_{k} k^{n+1}\left|a_{k}\right|^{2} r^{k-1}}{(B-A)-B \sum_{k=2}^{\infty} C_{k} k^{n+1}\left|a_{k}\right|^{2} r^{k-1}}\right\}<1 .
$$

So

$$
\sum_{k=2}^{\infty} k^{n+1} C_{k}(1+B)\left|a_{k}\right|^{2} r^{k-1} \leq B-A .
$$

and letting $r \rightarrow 1$

$$
\sum_{k=2}^{\infty} k^{n+1} C_{k}(1+B)\left|a_{k}\right|^{2} \leq B-A .
$$

Conversely, $f(z) \in V$ and satisfies (3). Since $r^{k-1}<1$, we have

$$
\begin{gathered}
\left.\left.\left|\sum_{k=2}^{\infty} k^{n+1}\right| a_{k}\right|^{2} z^{k-1} C_{k}\left|\leq \sum_{k=2}^{\infty} k^{n+1}\right| a_{k}\right|^{2} r^{k-1} C_{k} \\
\leq(B-A)-B \sum_{k=2}^{\infty} k^{n+1}\left|a_{k}\right|^{2} r^{k-1} C_{k} \\
\leq\left.\left|(B-A)+B \sum_{k=2}^{\infty} k^{n+1}\right| a_{k}\right|^{2} z^{k-1} C_{k} \mid
\end{gathered}
$$


Á. O. Páll-Szabó, O. Engel, E. Szatmári - Certain class of analytic functions ...

which gives (5) and hence follows that

$$
(1-\lambda)\left(\mathscr{S} \mathscr{R}^{n} f(z)\right)^{\prime}+\lambda\left(\mathscr{S} \mathscr{R}^{n+1} f(z)\right)^{\prime}=\frac{1+A w(z)}{1+B w(z)}
$$

that is $f(z) \in V P(n, \lambda, A, B)$.

Corollary 1. Let the function $f(z)$ defined by (1) be in the class $V P(n, \lambda, A, B)$. Then

$$
\left|a_{k}\right| \leq \sqrt{\frac{B-A}{k^{n+1} C_{k}(1+B)}},(k \geq 2) .
$$

The result (3) is sharp for the functions

$$
f(z)=z+\sqrt{\frac{B-A}{k^{n+1} C_{k}(1+B)}} e^{i \theta_{k}} z^{k},(k \geq 2) .
$$

\section{Distortion theorems}

Theorem 2. Let the function $f(z)$ defined by (1) be in the class $V P(n, \lambda, A, B)$. Then

$$
|z|-\sqrt{\frac{B-A}{2^{n+1} C_{2}(1+B)}}|z|^{2} \leq|f(z)| \leq|z|+\sqrt{\frac{B-A}{2^{n+1} C_{2}(1+B)}}|z|^{2} .
$$

The result is sharp.

Proof. We work with the technique used by Silverman [10]. We known that $\left|a_{k}\right| \leq$ $\sqrt{\frac{B-A}{2^{n+1} C_{2}(1+B)}}$ and $2^{n+1} C_{2} \leq k^{n+1} C_{k},(k \geq 2)$. Then

$$
\sum_{k=2}^{\infty}\left|a_{k}\right| \leq \sqrt{\frac{B-A}{2^{n+1} C_{2}(1+B)}}
$$

This way we have

$$
|f(z)| \leq|z|+\sum_{k=2}^{\infty}\left|a_{k}\right||z|^{k} \leq|z|+|z|^{2} \sum_{k=2}^{\infty}\left|a_{k}\right|,
$$


Á. O. Páll-Szabó, O. Engel, E. Szatmári - Certain class of analytic functions ...

so

$$
|f(z)| \leq|z|+\sqrt{\frac{B-A}{2^{n+1} C_{2}(1+B)}}|z|^{2} .
$$

Also, we have

$$
|f(z)| \geq|z|-\sum_{k=2}^{\infty}\left|a_{k}\right||z|^{k} \geq|z|-|z|^{2} \sum_{k=2}^{\infty}\left|a_{k}\right|
$$

So

$$
|f(z)| \geq|z|-\sqrt{\frac{B-A}{2^{n+1} C_{2}(1+B)}}|z|^{2} .
$$

The result is sharp for the function

$$
f(z)=z+\sqrt{\frac{B-A}{2^{n+1} C_{2}(1+B)}} e^{i \theta_{2}} z^{2},
$$

at $z= \pm|z| e^{-i \theta_{2}}$.

Corollary 2. Let the function $f(z)$ defined by (1) be in the class $V P(n, \lambda, A, B)$.

Then $f(z) \in U\left(0, r_{1}\right)$, where $r_{1}=1+\sqrt{\frac{B-A}{2^{n+1} C_{2}(1+B)}}$.

Theorem 3. Let the function $f(z)$ defined by (1) be in the class $V P(n, \lambda, A, B)$. Then

$$
1-\sqrt{\frac{B-A}{2^{n-1} C_{2}(1+B)}}|z| \leq\left|f^{\prime}(z)\right| \leq 1+\sqrt{\frac{B-A}{2^{n-1} C_{2}(1+B)}}|z| .
$$

The result is sharp.

Proof. We know that $k 2^{n+1} C_{2} \leq 2 k^{n+1} C_{k},(k \geq 2)$. Then

$$
\sum_{k=2}^{\infty} k\left|a_{k}\right| \leq 2 \sqrt{\frac{B-A}{2^{n+1} C_{2}(1+B)}}=\sqrt{\frac{B-A}{2^{n-1} C_{2}(1+B)}} .
$$

This way we have

$$
\left|f^{\prime}(z)\right| \leq 1+|z| \sum_{k=2}^{\infty} k\left|a_{k}\right| \leq 1+\sqrt{\frac{B-A}{2^{n-1} C_{2}(1+B)}}|z| .
$$


Á. O. Páll-Szabó, O. Engel, E. Szatmári - Certain class of analytic functions ...

So

$$
\left|f^{\prime}(z)\right| \geq 1-|z| \sum_{k=2}^{\infty} k\left|a_{k}\right| \geq 1-\sqrt{\frac{B-A}{2^{n-1} C_{2}(1+B)}}|z| .
$$

Corollary 3. Let the function $f(z)$ defined by (1) be in the class $V P(n, \lambda, A, B)$.

Then $f^{\prime}(z) \in U\left(0, r_{2}\right)$, where $r_{2}=1+\sqrt{\frac{B-A}{2^{n-1} C_{2}(1+B)}}$.

\section{Extreme points}

Theorem 4. Let the function $f(z)$ defined by (1) be in the class $V P(n, \lambda, A, B)$, with $\arg \left(a_{k}\right)=\theta_{k}$ where $2 \theta_{k}+(k-1) \delta \equiv \pi(\bmod 2 \pi), \forall k \geq 2$. Define

$$
f_{1}(z)=z
$$

and

$$
f_{k}(z)=z+\sqrt{\frac{B-A}{k^{n+1} C_{k}(1+B)}} e^{i \theta_{k}} z^{k},(k \geq 2 ; z \in U) .
$$

Then $f(z) \in V P(n, \lambda, A, B)$ if and only if $f(z)$ can expressed by $f(z)=\sum_{k=1}^{\infty} \mu_{k} f_{k}(z)$, where $\mu_{k} \geq 0$ and $\sum_{k=1}^{\infty} \mu_{k}=1$.

Proof. Assume that $f(z)=\sum_{k=1}^{\infty} \mu_{k} f_{k}(z)=z+\sum_{k=2}^{\infty} \sqrt{\frac{B-A}{k^{n+1} C_{k}(1+B)}} \mu_{k} e^{i \theta_{k}} z^{k}, \mu_{k} \geq 0$ and $\sum_{k=1}^{\infty} \mu_{k}=1$, then

$$
\sum_{k=2}^{\infty} \sqrt{\frac{k^{n+1} C_{k}(1+B)}{B-A}} \sqrt{\frac{B-A}{k^{n+1} C_{k}(1+B)}} \mu_{k}=\sum_{k=2}^{\infty} \mu_{k}=\left(1-\mu_{1}\right) \leq 1 .
$$

Hence $f(z) \in V P(n, \lambda, A, B)$. Conversly, let the function $f(z)$ defined by (1) be in the class $V P(n, \lambda, A, B)$, define

$$
\mu_{k}=\sqrt{\frac{k^{n+1} C_{k}(1+B)}{B-A}}\left|a_{k}\right|,(k \geq 2)
$$


Á. O. Páll-Szabó, O. Engel, E. Szatmári - Certain class of analytic functions ...

and

$$
\mu_{1}=1-\sum_{k=2}^{\infty} \mu_{k}
$$

From Theorem $1, \sum_{k=2}^{\infty} \mu_{k} \leq 1$ and so $\mu_{1} \geq 0$. Since $\mu_{k} f_{k}(z)=\mu_{k} z+a_{k} z^{k}$, then

$$
\sum_{k=1}^{\infty} \mu_{k} f_{k}(z)=z+\sum_{k=2}^{\infty} a_{k} z^{k}=f(z)
$$

Theorem 5. Let

$$
F(z)=I_{c} f(z)=\frac{c+1}{z^{c}} \int_{0}^{z} f(t) t^{c-1} d t, c>-1
$$

If $f \in V P(n, \lambda, 2 \alpha-1, B)$ then $F \in V P(n, \lambda, 2 \beta-1, B)$, where

$$
\beta=\beta(\alpha)=\frac{B+1}{2}-\frac{(c+1)^{2}(B-2 \alpha+1)}{2(c+2)^{2}} \geq \alpha .
$$

The result is sharp.

Remark: The operator $I_{c}$ is the well-known Bernardi operator.

Proof. Let $f \in V P(n, \lambda, 2 \alpha-1, B)$ and suppose it has the form (1). Then

$$
\begin{gathered}
F(z)=\frac{c+1}{z^{c}} \int_{0}^{z}\left(t+\sum_{k=2}^{\infty} a_{k} t^{k}\right) t^{c-1} d t= \\
=z+\sum_{k=2}^{\infty} \frac{c+1}{c+k} a_{k} z^{k}=z+\sum_{k=2}^{\infty} b_{k} z^{k} .
\end{gathered}
$$

Since $f \in V P(n, \lambda, 2 \alpha-1, B)$ we have

$$
\sum_{k=2}^{\infty} k^{n+1} C_{k}(1+B)\left|a_{k}\right|^{2} \leq B-(2 \alpha-1)
$$

or equivalently

$$
\frac{\sum_{k=2}^{\infty} k^{n+1} C_{k}(1+B)\left|a_{k}\right|^{2}}{B-2 \alpha+1} \leq 1 .
$$


Á. O. Páll-Szabó, O. Engel, E. Szatmári - Certain class of analytic functions ...

We know from Theorem 1 that $F \in V P(n, \lambda, 2 \beta-1, B)$ if and only if

$$
\sum_{k=2}^{\infty} k^{n+1} C_{k}(1+B)\left|b_{k}\right|^{2} \leq B-(2 \beta-1)
$$

or

$$
\frac{\sum_{k=2}^{\infty} k^{n+1} C_{k}(1+B)\left(\frac{c+1}{c+k}\right)^{2}\left|a_{k}\right|^{2}}{B-2 \beta+1} \leq 1 .
$$

We note that the inequalities

$$
\frac{k^{n+1} C_{k}(1+B)\left(\frac{c+1}{c+k}\right)^{2}\left|a_{k}\right|^{2}}{B-2 \beta+1} \leq \frac{k^{n+1} C_{k}(1+B)\left|a_{k}\right|^{2}}{B-2 \alpha+1}, \forall k \geq 2
$$

imply (13). From (14) we have

$$
\begin{gathered}
\frac{(c+1)^{2}}{(c+k)^{2}(B-2 \beta+1)} \leq \frac{1}{B-2 \alpha+1} \\
(c+1)^{2}(B-2 \alpha+1) \leq(c+k)^{2}(B-2 \beta+1), \forall k \geq 2 \\
\beta \leq \frac{B+1}{2}-\frac{(c+1)^{2}(B-2 \alpha+1)}{2(c+k)^{2}} .
\end{gathered}
$$

Let us consider the function

$$
E(x)=\frac{B+1}{2}-\frac{(c+1)^{2}(B-2 \alpha+1)}{2(c+x)^{2}},
$$

then its derivative is :

$$
E^{\prime}(x)=\frac{(c+1)^{2}(B-2 \alpha+1)}{(c+x)^{3}}>0 .
$$

$E(x)$ is an increasing function. In our case we need $\beta \leq E(k)$ and for this reason we choose $\beta=\beta(\alpha)=E(2)=\frac{B+1}{2}-\frac{(c+1)^{2}(B-2 \alpha+1)}{2(c+2)^{2}}$.

$$
\begin{gathered}
\beta(\alpha)>\alpha \Leftrightarrow B+1-\frac{(c+1)^{2}(B-2 \alpha+1)}{(c+2)^{2}}>2 \alpha \Leftrightarrow \\
\Leftrightarrow(c+2)^{2}>(c+1)^{2} .
\end{gathered}
$$


Á. O. Páll-Szabó, O. Engel, E. Szatmári - Certain class of analytic functions ...

The result is sharp, because if

$$
f_{2}(z)=z+\sqrt{\frac{B-2 \alpha+1}{2^{n+1} C_{2}(1+B)}} e^{i \theta_{2}} z^{2}
$$

then

$$
F_{2}=I_{c} f_{2}
$$

belongs to $V P(n, \lambda, 2 \beta-1, B)$ and its coefficients satisfy the corresponding inequality (3) with equality. Indeed,

$$
F_{2}(z)=z+\sqrt{\frac{B-2 \alpha+1}{2^{n+1} C_{2}(1+B)}} \frac{c+1}{c+2} e^{i \theta_{2}} z^{2}=z+\sqrt{\frac{B-2 \beta(\alpha)+1}{2 C_{2}(1+B)}} e^{i \theta_{2}} z^{2}
$$

and

$$
T\left(F_{2}\right)=2^{n+1} C_{2}(1+B) \frac{B-2 \beta(\alpha)+1}{2^{n+1} C_{2}(1+B)}=B-2 \beta(\alpha)+1 .
$$

Theorem 6. If $f \in V P(n, \lambda, A, B)$ then $F \in V P\left(n, \lambda, A^{*}, B\right)$, where $A^{*}=B-\frac{(c+1)^{2}(B-A)}{(c+2)^{2}}>A$. The result is sharp.

Proof. Let $f \in V P(n, \lambda, A, B)$ and suppose it has the form (1). Then

$$
F(z)=z+\sum_{k=2}^{\infty} \frac{c+1}{c+k} a_{k} z^{k}=z+\sum_{k=2}^{\infty} b_{k} z^{k}
$$

Since $f \in V P(n, \lambda, A, B)$ we have $\sum_{k=2}^{\infty} k^{n+1} C_{k}(1+B)\left|a_{k}\right|^{2} \leq B-A$ or equivalently

$$
\frac{\sum_{k=2}^{\infty} k^{n+1} C_{k}(1+B)\left|a_{k}\right|^{2}}{B-A} \leq 1
$$

We know from Theorem 1 that $F \in V P\left(n, \lambda, A^{*}, B\right)$ if and only if

$$
\frac{\sum_{k=2}^{\infty} k^{n+1} C_{k}(1+B)\left(\frac{c+1}{c+k}\right)^{2}\left|a_{k}\right|^{2}}{B-A^{*}} \leq 1, \forall k .
$$


Á. O. Páll-Szabó, O. Engel, E. Szatmári - Certain class of analytic functions ...

We note that

$$
\frac{k^{n+1} C_{k}(1+B)\left(\frac{c+1}{c+k}\right)^{2}\left|a_{k}\right|^{2}}{B-A^{*}} \leq \frac{k^{n+1} C_{k}(1+B)\left|a_{k}\right|^{2}}{B-A}
$$

implies (15). From (16) we have

$$
\begin{gathered}
\frac{(c+1)^{2}}{(c+k)^{2}\left(B-A^{*}\right)} \leq \frac{1}{B-A} \\
(c+1)^{2}(B-A) \leq(c+k)^{2}\left(B-A^{*}\right), \forall k \geq 2 \\
A^{*} \leq B-\frac{(c+1)^{2}(B-A)}{(c+k)^{2}} .
\end{gathered}
$$

Let us consider the function

$$
E(x)=B-\frac{(c+1)^{2}(B-A)}{(c+x)^{2}}
$$

its derivative is:

$$
E^{\prime}(x)=\frac{2(B-A)(c+1)^{2}}{(c+x)^{3}}>0
$$

$E(x)$ is an increasing function.

In our case we need $A^{*} \leq E(k), \forall k \geq 2$ and for this reason we choose

$A^{*}=E(2)=B-\frac{(c+1)^{2}(B-A)}{(c+2)^{2}}$.

We note that $A^{*}>A$, because

$$
B-A>\frac{(c+1)^{2}(B-A)}{(c+2)^{2}} \Leftrightarrow(c+2)^{2}>(c+1)^{2} .
$$

The result is sharp, because if

$$
f_{2}(z)=z+\sqrt{\frac{B-A}{2^{n+1} C_{2}(1+B)}} e^{i \theta_{2}} z^{2}
$$

then

$$
F_{2}=I_{c} f_{2}
$$

belongs to $\operatorname{VP}\left(n, \lambda, A^{*}, B\right)$ and its coefficients satisfy the corresponding inequality (3) with equality. Indeed, 
Á. O. Páll-Szabó, O. Engel, E. Szatmári - Certain class of analytic functions ...

$$
F_{2}(z)=z+\sqrt{\frac{B-A}{2^{n+1} C_{2}(1+B)}} \frac{c+1}{c+2} e^{i \theta_{2}} z^{2}=z+\sqrt{\frac{B-A^{*}}{2^{n+1} C_{2}(1+B)}} e^{i \theta_{2}} z^{2}
$$

and

$$
T\left(F_{2}\right)=2^{n+1} C_{2}(1+B) \frac{B-A^{*}}{2^{n+1} C_{2}(1+B)}=B-A^{*} .
$$

Theorem 7. If $f \in V P(n, \lambda, A, B)$ then $F \in V P\left(n, \lambda, A, B^{*}\right)$,where

$$
B^{*} \geq A+\frac{(A+1)(B-A)(c+1)^{2}}{(1+B)(c+2)^{2}-(B-A)(c+1)^{2}} .
$$

The result is sharp.

Proof. Let $f \in V P(n, \lambda, A, B)$ and suppose it has the form (1).

Since $f \in V P(n, \lambda, A, B)$ we have $\sum_{k=2}^{\infty} k^{n+1} C_{k}(1+B)\left|a_{k}\right|^{2} \leq B-A$ or equivalently

$$
\frac{\sum_{k=2}^{\infty} k^{n+1} C_{k}(1+B)\left|a_{k}\right|^{2}}{B-A} \leq 1 .
$$

We know from Theorem 1 that $F \in V P\left(n, \lambda, A, B^{*}\right)$ if and only if

$$
\sum_{k=2}^{\infty} k^{n+1} C_{k}\left(1+B^{*}\right)\left|a_{k}\right|^{2} \leq B^{*}-A
$$

or

$$
\frac{\sum_{k=2}^{\infty} k^{n+1} C_{k}\left(1+B^{*}\right)\left(\frac{c+1}{c+k}\right)^{2}\left|a_{k}\right|^{2}}{B^{*}-A} \leq 1 .
$$

We note that

$$
\frac{k^{n+1} C_{k}\left(1+B^{*}\right)\left(\frac{c+1}{c+k}\right)^{2}\left|a_{k}\right|^{2}}{B^{*}-A} \leq \frac{k^{n+1} C_{k}(1+B)\left|a_{k}\right|^{2}}{B-A}, \forall k
$$

implies (17).

The inequalities (18) are implied by

$$
\frac{(c+1)^{2}\left(1+B^{*}\right)}{(c+k)^{2}\left(B^{*}-A\right)} \leq \frac{(1+B)}{B-A}
$$


Á. O. Páll-Szabó, O. Engel, E. Szatmári - Certain class of analytic functions ...

$$
B^{*} \geq A+\frac{(A+1)(B-A)(c+1)^{2}}{(1+B)(c+k)^{2}-(B-A)(c+1)^{2}}, \forall k \geq 2 .
$$

Let

$$
E(x)=A+\frac{(A+1)(B-A)(c+1)^{2}}{(1+B)(c+x)^{2}-(B-A)(c+1)^{2}}
$$

its derivative is

$$
E^{\prime}(x)=\frac{-2(A+1)(c+1)^{2}(B-A)(1+B)(c+x)}{\left[(1+B)(c+x)^{2}-(B-A)(c+1)^{2}\right]^{2}}<0 .
$$

$E(x)$ is a decreasing function. In our case we need $E(k) \leq B^{*}$ and for this reason we choose

$$
\begin{gathered}
B^{*}=E(2)=A+\frac{(A+1)(B-A)(c+1)^{2}}{(1+B)(c+2)^{2}-(B-A)(c+1)^{2}} \\
B^{*}<B \Leftrightarrow(B-A)(c+1)(1+B)<(1+B)(c+2)(B-A) \Leftrightarrow c+1<c+2 .
\end{gathered}
$$

The result is sharp, because if

$$
f_{2}(z)=z+\sqrt{\frac{B-A}{2^{n+1} C_{2}(1+B)}} e^{i \theta_{2}} z^{2},
$$

then

$$
F_{2}=I_{c} f_{2}
$$

belongs to $\operatorname{VP}\left(n, \lambda, A, B^{*}\right)$ and its coefficients satisfy the corresponding inequality (3) with equality. Indeed,

$$
F_{2}(z)=z+\sqrt{\frac{B-A}{2^{n+1} C_{2}(1+B)}} \frac{c+1}{c+2} e^{i \theta_{2}} z^{2}=z+\sqrt{\frac{B^{*}-A}{2^{n+1} C_{2}\left(1+B^{*}\right)}} e^{i \theta_{2}} z^{2}
$$

and

$$
T\left(F_{2}\right)=2^{n+1} C_{2}\left(1+B^{*}\right) \frac{B^{*}-A}{2^{n+1} C_{2}\left(1+B^{*}\right)}=B^{*}-A .
$$


Á. O. Páll-Szabó, O. Engel, E. Szatmári - Certain class of analytic functions ...

\section{REFERENCES}

[1] A. Alb Lupas, Certain differential subordinations using Salagean and Ruscheweyh operators, Acta Universitatis Apulensis, 2012, Vol. 29, 125-129.

[2] H. S. Al-Amiri , On Ruscheweyh derivatives, Ann. Polon. Math., 38(1980), $87-94$.

[3] A. A. Attiya and M. K. Aouf, A study on certain class of analytic functions defined by Ruscheweyh derivative, Soochow J. Math., 33(2)(2007), 273-289.

[4] H. M. Hossen, G. Ş. Salagean, M. K. Aouf, Notes on certain classes of analytic functions with negative coefficients, Mathematica(Cluj), 39(62) no.2(1997), 165-179.

[5] P. T. Mocanu, T. Bulboacă, G. S. Sălăgean, The geometric theory of univalent functions, Casa Cărţii de Ştiinţă, Cluj-Napoca, 1999.

[6] Á. O. Páll-Szabó, O. Engel, Certain class of analytic functions with varying arguments defined by Salagean derivative, Proceedings of the 8th International Conference on Theory and Applications of Mathematics and Informatics,ICTAMI 2015, Alba Iulia, Romania,17th-20th of September,2015, 113-120.

[7] St. Ruscheweyh, New criteria for univalent functions, Proc. Amer. Math. Soc., 49(1975), 109-115.

[8] G. S. Sălăgean, Subclasses of univalent functions, Lecture Notes in Math. (Springer Verlag), 1013(1983), 362-372.

[9] G. S. Sălăgean, Integral properties of certain classes of analytic functions with negative coefficients, Int. Journal of Math. and Math. Sci., 1(2005), 125-131.

[10] H. Silverman, Univalent functions with varying arguments, Houston J. Math., 17(1981), 283-287.

Ágnes Orsolya Páll-Szabó

Babeş-Bolyai University, Cluj-Napoca, Romania

email: pallszaboagnes@math.ubbcluj.ro

Olga Engel

Babeş-Bolyai University, Cluj-Napoca, Romania

email: engel_olga@hotmail.com

Eszter Szatmári

Babeş-Bolyai University, Cluj-Napoca, Romania

email: szatmari.eszter@math.ubbcluj.ro 